\title{
Efeitos da cinesioterapia através do método de facilitação neuromuscular proprioceptiva no equilíbrio e na capacidade funcional de idosos
}

\author{
Effects of kinesiotherapy through the proprioceptive neuromuscular facilitation method in \\ equilibrium and functional capacity of elderly
}

Efectos de la kinesioterapia a través del método de facilitación neuromuscular propietario en equilibrio y capacidad funcional de ancianos

Katiele Silva dos Santos Lima ${ }^{1 *}$, Luis Rodolfo Teixeira Santos ${ }^{1}$, Ricardo João Soares Barros Filho².

\section{RESUMO}

Objetivos: Analisar os efeitos da cinesioterapia através do método de Facilitação Neuromuscular Proprioceptiva no equilíbrio e na capacidade funcional de idosos. Métodos: Trata-se de um estudo quantitativo, tipo intervencional, transversal. Aplicou-se aos participantes da pesquisa a escala de equilíbrio de Berg (EEB) e o teste Timed get up and go, em seguida, os participantes foram submetidos a aplicação do método de Facilitação Neuromuscular Proprioceptiva aos membros superiores e inferiores. Aplicado contra a resistência manual do fisioterapeuta em três séries de 10 repetições para cada membro uma vez ao dia por um período de dez dias. Ao final do tratamento, aplicou-se novamente a EEB e o teste Timed get up and go, comparando-se os resultados finais com aqueles obtidos antes do tratamento com o FNP. Resultados: Observou-se que os valores obtidos na EEB na pré-terapia com o método FNP apresentavamse baixos, o que significa dizer que os participantes apresentavam déficits motores. Após a intervenção com o método FNP, foi novamente aplicada a EEB. A mesma apresentou valores diferentes daqueles obtidos anteriormente, sendo estes valores maiores. Bem como uma diminuição no tempo de realização do teste Timed get up and go. Conclusão: Conclui-se então, que a utilização do método FNP em idosos influenciou positivamente no ganho de equilíbrio e no aumento da capacidade funcional.

Palavras-chave: Terapia por exercício, Assistência a Idosos, Métodos.

\begin{abstract}
Objectives: To analyze the effects of kinesiotherapy through the Proprioceptive Neuromuscular Facilitation method on the balance and functional capacity of the elderly. Methods: This is a quantitative, interventional, cross-sectional study. Toe Berg balance scale (BSE) and the Timed get up and go test were applied to the participants, Participants underwent the Proprioceptive Neuromuscular Facilitation method to the upper and lower limbs. Applied against manual resistance of the physiotherapist in three sets of 10 repetitions for each member once a day for a period of ten days. At the end of the treatment, BSE and the Timed get up and go test were applied again, comparing the final results with those obtained prior to PNF treatment. Results: It was observed that the values obtained in BSE in the pre-therapy with the PNF method were low, which means that the participants had motor deficits. After the intervention with the FNP method, BSE was again applied. The same presented values different from those obtained previously, being these values greater. As well as a decrease in timing of the Timed get up and go test. Conclusion: It was concluded that the use of the FNP method in the elderly positively influenced the balance gain and the increase in functional capacity.
\end{abstract}

Key words: Exercise Therapy, Elderly Care, Methods.

${ }^{1}$ Faculdade Mauricio de Nassau, Campus Redenção Teresina, Piauí. *E mail: katiele.fisioterapia@gmail.com

${ }^{2}$ Fisioterapeuta Intensivista, Hospital de Urgências de Teresina, Piauí. 


\section{RESUMEN}

Objetivos: Analizar los efectos de la kinesioterapia a través del método de Facilitación Neuromuscular Propioceptiva sobre el equilibrio y la capacidad funcional de las personas de edad avanzada. Métodos: Este es un estudio cuantitativo, intervencionista y transversal. la escala de equilibrio de Berg (BSE) y la prueba de levantarse y pasar cronometrada se aplicaron a los participantes. Los participantes se sometieron al método de facilitación neuromuscular propioceptiva en las extremidades superiores e inferiores. Se aplica contra la resistencia manual del fisioterapeuta en tres series de 10 repeticiones para cada miembro una vez al día durante un período de diez días. Al final del tratamiento, se aplicaron nuevamente la BSE y la prueba de início y uso de Timed, comparando los resultados finales con los obtenidos antes del tratamiento con FNP. Resultados: Se observó que los valores obtenidos en la EEB en la terapia previa con el método de FNP eran bajos, lo que significa que los participantes tenían déficits motores. Después de la intervención con el método FNP, se aplicó nuevamente la EEB. Los mismos valores presentados difieren de los obtenidos previamente, siendo estos valores mayores. Así como una disminución en el tiempo de la prueba de levantarse e ir cronometrada. Conclusión: Se concluyó que el uso del método FNP en ancianos influye positivamente en la ganancia de equilibrio y en el aumento de la capacidad funcional.

Palabras clave: Terapia de ejercicio, Cuidado de ancianos, Métodos.

\section{INTRODUÇÃO}

O envelhecimento da população brasileira tem se destacado por seu crescimento acelerado nos últimos anos, constituindo assim o segmento populacional que mais cresce atualmente no país (MINISTÉRIO DA SAÚDE, 2010).

Segundo dados do Instituto Brasileiro de Geografia e Estatística (IBGE) pessoas com idade igual ou superior a 60 anos, representam cerca de $10 \%$ da população Brasileira. Já segundo a Organização Mundial de Saúde (OMS) até o ano de 2025, estima-se que a população idosa no Brasil deverá ter aumentado em até quinze vezes, colocando assim, o Brasil na sexta posição mundial quanto ao contingente de idosos (MINISTÉRIO DA SAÚDE, 2010). É importante associar a este fato, que com o avanço da idade, aumentam também as limitações cognitivas e funcionais, bem como a elevação do índice de doenças crônico degenerativas, levando essas pessoas a uma maior dependência na realização de atividades cotidianas (FIEDLER MM e PERES KG, 2008).

As limitações em pessoas idosas são de etiologia multifatorial. É possível observar uma relação mútua entre fatores intrínsecos, ambientais e comportamentais. Caracterizam-se como fatores intrínsecos, as alterações fisiológicas características do próprio processo de envelhecimento, conjuntamente aos processos patológicos, além da influência dos fatores psicológicos e medicamentosos (GAZZOLA JM, et al., 2004). Para os idosos que possuem algum comprometimento funcional do equilíbrio, a reabilitação precoce objetiva prevenir a evolução do mesmo e recuperar a perda funcional incipiente, amenizando ou evitando assim, que limitações funcionais possam progredir e culminar em quedas, cujas consequências principais são incapacidade e morte (GAZZOLA JM, et al., 2004).

A Facilitação Neuromuscular Proprioceptiva é uma técnica de alongamento e fortalecimento utilizada para melhorar a elasticidade dos músculos e tem se mostrado com um efeito positivo sobre a movimentação ativa e passiva. Pesquisas recentes têm demonstrado a eficácia dessa intervenção no pico de torque e na força muscular (SILVA MLG, et al., 2013). Os procedimentos básicos da Facilitação Neuromuscular Proprioceptiva fornecem ao terapeuta as ferramentas necessárias para ajudar os seus pacientes a atingir uma função motora eficiente (SOUZA IMB, et al., 2015).

É importante entender o processo de envelhecimento em suas diferentes fases, com o objetivo de garantir o esclarecimento para esses indivíduos e propiciar uma diminuição nas taxas de morbidade e mortalidade. Frente a isso, o método de Facilitação Neuromuscular proprioceptiva se apresenta como uma forma de tratamento promissora no que diz respeito ao ganho de força e equilíbrio em idosos, afetando 
assim diretamente o número de casos de perda de autonomia e dependência. $\mathrm{O}$ trabalho teve como objetivo avaliar os efeitos da cinesioterapia através do método de facilitação Neuromuscular Proprioceptiva no equilíbrio e na capacidade funcional de idosos.

\section{MATERIAIS E MÉTODOS}

Trata-se de um estudo quantitativo, tipo intervencional, transversal. Este trabalho foi aprovado pelo comitê de ética em pesquisa do hospital de urgências de Teresina - HUT (protocolo CEP $\mathrm{N}^{\circ} 20 / 16$ ). A população foi constituída por pacientes que se encontravam internados em um hospital de referência em atendimentos de urgência e emergência em Teresina - Piauí, e todos participantes idosos. A amostra foi constituída de cinco indivíduos selecionados por conveniência. Foi aplicada aos participantes da pesquisa a Escala de Equilibro de Berg - EEB, que visa avaliar o equilíbrio e força muscular que ambos possuem, e o teste Timed get up and go, que ajuda a identificar o grau de dependência funcional que os mesmos possuem. Em seguida, os participantes foram submetidos à aplicação do método FNP (Facilitação Neuromuscular Proprioceptiva), o mesmo foi aplicado aos membros inferiores e superiores, através do padrão flexor e extensor.

Os critérios de inclusão para o presente estudo foram: Participantes com faixa etária igual ou acima de 65 anos, bom desempenho cognitivo e capacidade funcional para realização dos testes propostos. Os critérios de exclusão foram: Pacientes com histórico de fraturas em membros inferiores, portadores de patologias neurológicas que levem a alterações do equilíbrio, cadeirantes e pacientes com diagnóstico de labirintopatias e cardiopatias com baixa fração de ejeção.

Os movimentos foram realizados contra a resistência manual do terapeuta, em três séries de 10 repetições para cada membro uma vez ao dia por um período de dez dias. Ao final dos dez dias de aplicação do método, aplicou-se novamente a EEB e o teste Timed get up and go, comparando-se os resultados finais com aqueles obtidos antes do tratamento com o FNP.

Para análise estatística dos dados foi utilizado o Microsoft Excel. Optou-se por uma análise de estatística descritiva dos resultados.

\section{RESULTADOS E DISCUSSÃO}

O presente estudo foi composto por uma amostra de 5 participantes, sendo destes, 2 do sexo masculino e 3 do sexo feminino, com uma faixa etária entre 69 e 80 anos para o sexo masculino e entre 65 e 79 anos para o sexo feminino. Essa faixa etária foi determinada, uma vez que, segundo o Ministério da saúde (2006), no Brasil, é definida como idosa a pessoa que tem 60 anos ou mais de idade.

Os distúrbios do equilíbrio aumentam consideravelmente com o avanço da idade, isto ocorre principalmente pela redução na qualidade da regulação postural, que consequentemente são acompanhados por um maior risco de quedas (CRUZ RM e TOUGUINHA HM, 2015).

Segundo Fiedler MM e Peres KG (2008), é definido como capacidade funcional o potencial que os idosos apresentam para decidir e atuar em suas vidas de forma independente.

No estudo foi observado que os valores obtidos na EEB na pré-terapia com o método FNP apresentavam-se baixos, o que significa dizer que os participantes apresentavam déficits motores, principalmente na manutenção do equilíbrio durante a realização das posturas e funções propostas pela EEB, que é composta com 14 itens, que envolvem tarefas funcionais especificas em diferentes bases de apoio. Cada item corresponde a um escore de 0 a 4.

Os pontos são obtidos através do desempenho do indivíduo em relação a cada tarefa, que envolvem posições a serem mantidas, alcance, equilíbrio, entre outros, sendo respectivamente, o menor valor considerado como um mau desempenho e o maior valor como um ótimo desempenho, ou seja, dentro dos padrões da normalidade. 
Após a intervenção com o método FNP no período de 10 dias, foi novamente aplicada a EEB. A mesma apresentou valores diferentes daqueles obtidos na pré-intervenção, sendo estes valores maiores. Os participantes apresentaram uma melhora considerável na desenvoltura das atividades e funções propostas.

Os principais ganhos posturais e funcionais dos participantes do estudo, analisados através da EEB estão demonstrados no Gráfico 1.

Gráfico 1 - Principais ganhos posturais e funcionais observados através da EEB.

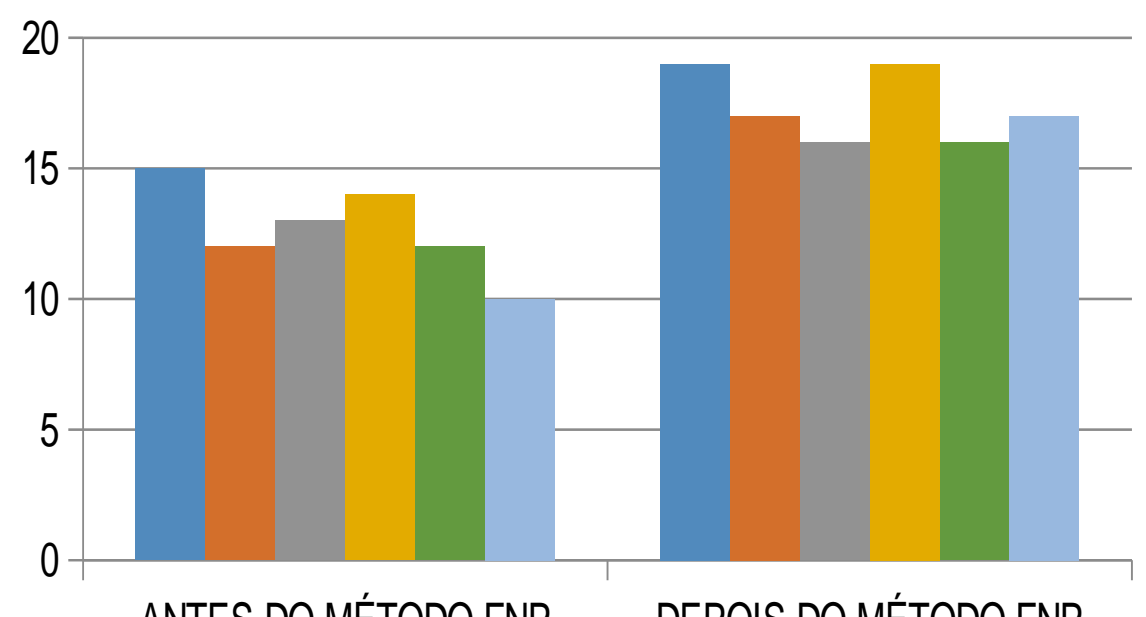

Em pé para sentado $(26,6 \%)$

- Sentado para em pé $(41,6 \%)$

- Transferências (23,8\%)

Permanecer em pé com os pés juntos (35,7\%)

- Girar $360^{\circ}(33,3 \%)$

Em pé sem apoio com um pé na frente $(70, \%)$

Fonte: Lima KSS, Santos LRT, Filho RJSB, 2019.

Esses ganhos correspondem principalmente às posições de em pé para sentado, onde no período de pré-intervenção observou-se que os participantes utilizavam a parte posterior dos membros inferiores e as mãos para controlar a descida. Após a intervenção, os ganhos foram significativos, onde se pôde observar que os participantes eram capazes de controlar a descida até a cadeira e fazer um uso mínimo das mãos. Estatisticamente observa-se que de uma forma geral houve um ganho de $26,67 \%$. Na posição de sentado para de pé, na pré-intervenção observou-se que os participantes eram capazes de levantar utilizando as mãos, e ao ficar de pé, necessitavam de alguma ajuda para estabilizar-se. Após a intervenção, os mesmos foram capazes de ficar em pé, com o uso mínimo das mãos e estabilizarem-se independentemente, apresentando um percentual de melhora de $41,67 \%$.

No caso das transferências, na pré-intervenção, os participantes necessitaram de ajuda do avaliador, orientação verbal e o uso das mãos para completar a função. Após a intervenção, os mesmos realizaram as transferências apenas com comandos verbais e uso mínimo das mãos, apresentando um percentual de melhora de $23,08 \%$. Na posição de pé com os pés juntos, observou-se que todos os participantes foram capazes de permanecer em pé com os pés juntos e manter o equilíbrio por no máximo 30 segundos, sob supervisão. Após a intervenção, os mesmos já eram capazes de posicionar os pés juntos independentemente e manter o equilíbrio com segurança por 1 minuto, apresentando um percentual de melhora de $35,71 \%$.

No movimento de girar $360^{\circ}$, os participantes mostraram-se capazes de girar $360^{\circ}$, porém lentamente e sob supervisão devido ao risco de queda. Após a intervenção, os mesmos foram capazes de realizar os movimentos em até 4 segundos, sob supervisão, apresentando um percentual de melhora de $33,33 \%$. $\mathrm{Na}$ realização do movimento de ficar em pé, sem apoio, com um pé na frente, na pré-intervenção, os participantes mostraram-se capazes de dar um pequeno passo, independentemente, sob supervisão e 
permanecer por até 30 segundos. Após a intervenção, os mesmos foram capazes de colocar um pé a frente do outro, com segurança e permanecer por 30 segundos, obtendo assim, um percentual de melhora significativamente relevante de $70,00 \%$.

Os resultados positivos obtidos neste estudo corroboram com o estudo realizado por Santos TB, et al. (2012), que realizou um estudo com uma amostra de 4 indivíduos com idades entre 65 e 80 anos. Este estudo teve como objetivo a análise através da CIF (Classificação Internacional da Funcionalidade) do ganho funcional dos participantes após a intervenção com o método FNP. Ele concluiu que a aplicação do método resultou em importante melhora do quadro clinico e dos aspectos cinético funcionais dos participantes. Segundo ele, isso foi possível porque o método FNP trabalha simultaneamente com o ganho de mobilidade, treino de atos motores e o equilíbrio, fatores que, com o uso de outras técnicas, são alcançados isoladamente.

Silva MLG, et al. (2013) alcançou resultados positivos em um estudo realizado com uma amostra de 11 participantes, do sexo feminino, com faixa etária entre 60 e 80 anos. Segundo ele, o tratamento com FNP obtém a máxima quantidade de atividade que pode ser conseguida em cada esforço voluntário e o maior número possível de repetições desta atividade para facilitar a resposta. Zipperer A e Brunn G (2011) afirmam nos resultados de seu estudo, realizado com uma amostra de 27 idosas, com o objetivo de ganho de equilíbrio e capacidade funcional, que o FNP propiciou ganhos na qualidade de movimentação requerida nas atividades motoras da vida diária bem como o aumento na disposição na realização de tais atividades. Já Carvalho KL, et al. (2008) relata que a aplicação do método FNP em idosos resulta em uma melhora da mobilidade e da funcionalidade do idoso, podendo assim, adiar a necessidade de um cuidador. Ressalta ainda, outro fator que torna o método eficaz, que é a facilidade de aplicação do mesmo, que necessita apenas da habilidade do fisioterapeuta.

O Gráfico 2 mostra o tempo utilizado por cada participante para a realização do teste Timed get up and go.

Gráfico 2 - Tempo utilizado para realização do teste em segundos.



Participante $1(34,4 \%)$

- Participante 2 (32\%)

Participante $3(31,2 \%)$

Participante 4 (24\%)

Participante 5 (33,3\%)

Fonte: Lima KSS, Santos LRT, Filho RJSB, 2019. 
A pontuação do teste mostra uma grande relação com o equilíbrio, velocidade da marcha e capacidade funcional. A proposta de avaliação do teste está relacionada com seu tempo de execução. Tempos reduzidos indicam uma melhor independência quanto à mobilidade, diminuindo a possibilidade de quedas. O teste Timed Get up and go é um teste que avalia o nível de mobilidade funcional de forma rápida e pratica e consiste em mensurar em segundos o tempo gasto por um indivíduo para levantar de uma cadeira, andar uma distância de 3 metros, dar a volta, retornar a cadeira e sentar novamente (WOELLNER SS, et al., 2014). Ainda segundo o autor, indivíduos adultos independentes e sem alterações no equilíbrio, realizam o teste em 10 segundos ou menos, os que são dependentes em transferências básicas, realizam o teste em 20 segundos ou menos e os que necessitam de mais de 20 segundos para realizar o teste são dependentes em muitas atividades da vida diária e na mobilidade.

Analisando o gráfico 2, é possível observar que os participantes 1, 2 e 4 apresentaram respectivamente os tempos de 29,25 e 25 segundos para a realização do teste no período pré-intervenção. Esses valores classificam os participantes citados como indivíduos que possuem um médio risco para quedas e nível médio de capacidade para a realização de atividades funcionais. Após a intervenção, analisando novamente esses pacientes, observou-se que os mesmos utilizaram um tempo menor para a realização do teste, sendo o tempo de 19, 17 e 19 segundos respectivamente, o que os classifica como indivíduos que evoluíram para um baixo risco para quedas e uma boa capacidade para a realização de atividades funcionais. Estatisticamente, esses participantes apresentaram uma redução no tempo de realização do teste de $34,48 \%, 32,00 \%$ e $24,00 \%$ respectivamente.

Em relação aos pacientes 3 e 5, é possível observar que os mesmos apresentaram no período pré intervenção tempos de 32 e 30 segundos respectivamente, o que os classificou como indivíduos que apresentam um alto risco para quedas e déficits para realizar suas atividades funcionais. Após a intervenção, observou-se que esses pacientes tiveram uma melhora significativa no desempenho do teste e consequentemente realizando o mesmo em um tempo menor. Os mesmos apresentaram um tempo de $22 \mathrm{e}$ 20 segundos respectivamente, o que passou a classifica-los como indivíduos de médio risco para quedas e com média capacidade para o desempenho das suas atividades funcionais. Estatisticamente, esses participantes apresentaram uma redução no tempo de realização do teste de $31,25 \%$ e $33,33 \%$ respectivamente.

De um modo geral, todos os 5 participantes apresentaram, antes da intervenção com o método FNP, um tempo médio de realização do teste de $28,2 \%$, e após a intervenção, um tempo médio de $19,4 \%$, apresentando dessa forma, uma redução do tempo médio total dos participantes de $31,21 \%$.

Os participantes do estudo também apresentaram, após serem submetidos ao método FNP, melhoras em relação à marcha, tendo como principais ganhos a velocidade da marcha, balanço de tronco e membros e habilidade para retornar.

Os resultados positivos encontrados, vão de acordo com o que relatou Lira VA e Araújo CGS (2000) em seus estudos, pois segundo ele, é considerado que o Teste timed get up and go pode medir diretamente o desempenho funcional de pessoas idosas, sendo assim, não se deve ter dúvidas quanto a sua validade lógica. Destaca ainda, que a utilização do teste em consultórios de profissionais da saúde pode consistir em uma estratégia útil, para identificar indivíduos que possuem maiores limitações no desempenho de atividades funcionais.

Em um estudo realizado por Rodrigues JE, et al. (2010) que teve como objetivo analisar o ganho de equilíbrio após a intervenção com o método FNP, utilizando como parâmetro de avaliação o teste Timed get up and go, o mesmo afirma que os resultados encontrados revelaram uma diminuição estatisticamente significante na média do tempo de realização do teste após a aplicação do método FNP nos membros inferiores de idosas sentarias. Corroborando assim com os resultados encontrados no presente estudo.

Existem na literatura outros estudos que assim como o presente trabalho objetivaram a melhora do equilíbrio e da capacidade funcional de idosos, porém, utilizando outros métodos de intervenção fisioterapêutica. No estudo realizado por Leal SMO, et al. (2010), foi analisado o equilíbrio postural e a 
autonomia funcional em uma amostra de 42 idosas, com idades entre 60 e 85 anos. As participantes do estudo realizaram um programa baseado em treino com exercícios resistidos, em um período de 12 semanas, com uma frequência de duas vezes na semana e 50 minutos de duração. Apesar da desistência de grande parte da amostra do estudo, ao final do trabalho o autor afirma que o treinamento aplicado atingiu os objetivos propostos pela pesquisa.

Lopes MNSS, et al. (2010), realizou um estudo com uma amostra de 7 idosos, com o objetivo de analisar o ganho de equilíbrio após a aplicação de um protocolo fisioterapeutico contendo exercícios metabólicos para aquecimento, seguido de alongamento e exercícios de fortalecimento e treino de marcha e equilíbrio, realizadas 2 vezes por semana, com duração de 50 minutos cada, por um período de 2 meses. $O$ autor afirma que após o início das intervenções três participantes apresentaram quadro de dor muscular e foram encontrados sintomas como cefaleia, dor em região dorsal e membros inferiores e sugere que novos estudos sejam realizados com uma amostra maior.

Bruni BM, et al. (2008), realizou um estudo com uma amostra de 25 participantes idosas, para avaliar o ganho de equilíbrio e capacidade funcional após 10 sessões de hidroterapia 1 vez por semana com duração de 40 minutos durante 10 semanas. Atividades como caminhadas na piscina, alongamento, fortalecimento e treino de equilíbrio postural foram abordados no estudo. Ao final do estudo os autores afirmam ter alcançado resultados positivos, porém sugerem que novos estudos baseados no mesmo tema sejam realizados com um tempo de intervenção maior.

\section{CONCLUSÃO}

Conclui-se então, que a utilização do método FNP em idosos influenciou positivamente no ganho de equilíbrio e no aumento da capacidade funcional, oferecendo, por conseguinte, um maior nível de mobilidade e uma menor propensão a quedas na população estudada. Sugere-se a realização de novos estudos com o tema, envolvendo amostras maiores, fazendo uso de grupo controle para que se possa definir com mais precisão a influência do FNP no ganho de equilíbrio e capacidade funcional em idosos.

\section{REFERÊNCIAS}

1- BIASOLI MC. Tratamento Fisioterápico na Terceira Idade. Revista Bras Med. Vol. 64, Edição Especial, p. 62-68, 2007.

2- BRASIL, Ministério da Saúde. Secretaria de Atenção à Saúde. Departamento de Ações Programáticas e estratégicas. Atenção à saúde da pessoa Idosa e envelhecimento. Área técnica saúde do Idoso. Brasília, Vol. 12, p.11, 2010.

3- BRUNI BM, et al. Avaliação do equilíbrio postural em idosos praticantes de Hidroterapia em grupo. O mundo da saúde São Paulo. São Paulo, Vol. 32, n.1, p.56-63, 2008.

4- CARVALHO KL, et al. O método Kabat no tratamento Fisioterapêutico da doença de Alzheimer. Revista Kairós. São Paulo, Vol.11, n.2, p.181-195, 2008.

5- CRUZ RM, TOUGUINHA H. Efeitos do treinamento funcional na melhora das atividades da vida diária de idosos. Rev. Saúde em foco. São Paulo, n.7, 2015.

6- DUARTE J, HELFSTEIN TT. Estudo comparativo das técnicas de Cinesioterapia respiratória convencional e associado as diagonais de membros superiores na reexpansão torácica em Indivíduos adultos jovens. J Health Science Institute. São Paulo, Vol. 29, n.3, p.198-201, 2011.

7- FIEDLER MM.; PERES, KG. Capacidade Funcional e Fatores associados em Idosos do sul do Brasil. Cad. Saúde Pública. Rio de Janeiro, Vol. 24, n.2, p.409-415, 2008.

8- GAMA ZAS, et al. Influência da Frequência de Alongamento utilizando a Facilitação Neuromuscular Proprioceptiva na flexibilidade dos músculos Isquiostibiais. Revista Bras Med Esporte. Cabezo de Torres, Vol. 13, n.1, p.33-38, 2007.

9- GAZZOLA JM, et al. Caracterização Funcional do Equilíbrio de Idosos em serviço de Reabilitação Gerontológica. Rev. Fisioter. Univ. São Paulo. São Paulo, Vol. 11, n.1, p.1-14, 2004.

10- LEAL SMO, et al. Efeitos do treinamento funcional na autonomia funcional, equilíbrio e qualidade de vida de idosas. R. Bras. e mov. São Paulo, Vol. 17, n.3, p. 61-69, 2010. 
11- LIRA VA, ARAÚJO CGS. Teste de sentar-se e levantar: Estudos de fidedignidade. Rev. Bras. Ciên. E Mov. Rio de Janeiro, Vol. 8, n.2, p. 9-18, 2000.

12- LOPES MNSS, et al. Eficácia de um protocolo fisioterapêutico para equilíbrio em idosos institucionalizados. Ciências biológicas e da saúde. Londrina, Vol. 31, n.2, p.143-152, 2010.

13- MORTARI DM, et al. Efeitos da Crioterapia e Facilitação Neuromuscular Proprioceptiva sobre a força muscular nas musculaturas flexora e extensora de joelho. Fisioterapia e Pesquisa. São Paulo, Vol. 16, n.4, p.329-334, 2009.

14- MILESKI ME, et al. Efeitos da Eletroestimulação e da Facilitação Neuromuscular Proprioceptiva na Marcha de Hemiparéticos. Revista Ciência e Saúde. Porto Alegre, Vol. 6, n.1, p.29-36, 2013.

15- MARQUES WV, et al., Influencia da capacidade funcional no risco de quedas em adultos com Artrite Reumatóide. Rev Bras Reumatol. Goiás, Vol. 54, n.5, p.404-408, 2014.

16- RODRIGUES JE, et al. O uso da Facilitação Neuromuscular Proprioceptiva em membros inferiores para ganho de equilíbrio dinâmico em Idosas sedentárias. Fisioterapia Ser. São Paulo, Vol. 5, n.4, p.230-234, 2010.

17- SILVA MLG, et al. Efeito dos exercícios de Facilitação Neuromuscular Proprioceptiva de tronco na Estabilidade lombar em Idosos. Terapia Manual. Teresina, Vol. 11, n.53, p.348-354, 2013.

18- SANTOS TB, et al. Facilitação Neuromuscular Proprioceptiva na Doença de Parkinson: Relato de Eficácia Terapêutica. Fisioterapia e Movimento. Curitiba, Vol. 25, n.2, p.281-289, 2012.

19- SOUZA IMB, et al. Alterações Musculares em Jovens Submetidas a Treinamento Baseado em Facilitação Neuromuscular Proprioceptiva (FNP) Vistas por Análise Eletromiográfica Superficial e Dinamométrica. Amazonas: FAPEAM, 2015.

20- STORTI LB, et al. Fragilidade de Idosos Internados na Clínica Médica na Unidade de Emergência de Um Hospital Geral Terciário. Texto Contexto Enfermagem. Florianópolis, Vol. 22, n.2, p.552-559, 2013.

21- SOARES MA, SACCHELLI T. Efeitos da Cinesioterapia no Equilíbrio de Idosos. Revista Neurocienc. São Paulo, Vol. 16, n.2, p.97-100, 2008.

22- TAVARES AC, SACCHELLI T. Avaliação da atividade Funcional em Idosos submetidos a Cinesioterapia em solo. Revista Neurocienc. São Paulo, Vol. 17, n.1, p.19-23, 2009.

23- WOELLNER SS, et al. Protocolos de equilíbrio e quedas em Idosos. Neurociências. Santa Catarina, Vol.10, n.2, 2014.

24- ZIPPERER A, BRUN G. Efeitos do Alongamento Ativo e do Método de Facilitação Neuromuscular Proprioceptiva. Revista Ágora. Vol. 18, n.1. 2011. 